Gray, W. D., Schoelles, M. J., Bringsjord, S. A., Burrows, K., \& Colder, B. (2003). Sage: Five powerful ideas for studying and transforming the intelligence analyst's task environment, Human Factors and Ergonomics Society (pp. 1019-1023). Santa Monica, CA: Human Factors and Ergonomics Society.

p. 1019

\title{
SAGE: FIVE POWERFUL IDEAS FOR STUDYING AND TRANSFORMING THE INTELLIGENCE ANALYST'S TASK ENVIRONMENT
}

\author{
Wayne D. Gray, Michael J. Schoelles, Selmer Bringsjord \\ Rensselaer Polytechnic Institute \\ [grayw/brings/schoem]@rpi.edu
}

\author{
Kirk Burrows, \& Brian Colder \\ Booz Allen Hamilton \\ [burrows_kirk/colder_brian]@bah.com
}

\begin{abstract}
Sage provides a scaled world in which real and simulated intelligence analysts work to solve realistic scenarios in innovative task environments. All aspects of Sage are instrumented for data collection and Sage itself is built to facilitate the swapping in and out of prototypes of innovative tools for information search, hypothesis generation, and hypothesis testing. Sage focuses not simply on the promised functionality of these tools, but the way in which the design of the interface supports or hinders the realization of this functionality. Beyond the individual tool and its interface, Sage supports the cognitive engineering of integrated task environments by the use of simulated cyborgs (simBorgs). SimBorgs combine high-fidelity computational cognitive models with low-fidelity artificial intelligence (AI) based reasoning components. This combination of cognitive modeling with AI enables the creation of intelligent agents, simBorgs, that will work tirelessly to perform usability testing on various combinations of tasks and interfaces.
\end{abstract}

NIMD supports innovative, creative, and high-risk research with the goal of supporting the intelligence analyst (IA) in discovering "novel intelligence from massive data". In advancing towards this goal, most of the funded projects focus on building software tools that can be used to augment the intelligence analyst's (IA's) task environment. Sage takes a different path toward this goal, one that may support some of the other efforts while contributing innovative and creative research of its own.

Sage is the vision of Booz Allen Hamilton (Booz Allen) which teamed with Planet 9 Studios and Rensselaer Polytechnic Institute to help make the vision happen. Although the current report encompasses the full scope of Sage it emphasizes the work done by Rensselaer with a strong emphasis on the cognitive engineering focus provided by Rensselaer's CogWorks Laboratory.

Sage is built around five powerful ideas. Each of the following sections emphasizes one of these powerful ideas. The final section summarizes these ideas and accesses our likelihood of achieving them.

\section{Powerful Idea \#1: Sage as a Scaled World}

"In field research there is too much [complexity] to allow for any more definite conclusions, and in laboratory research, there is usually too little complexity to allow for any interesting conclusions". (Brehmer \& Dörner, 1993 p. 172).

What's a researcher to do? Traditional laboratory

\footnotetext{
${ }^{1}$ Not an acronym.
}

studies are unlikely to make timely contributions to pressing national issues, but the daily work environment of an intelligence analyst consists of tasks, problems, and solutions that entail a myriad of unique circumstances, facts, and happenstance. Furthermore, the community of IAs is a diverse community of problem solvers. Individual IAs approach task problems with different analytical methods and knowledge. This combination of extreme heterogeneity of the subject population and extreme heterogeneity of the tasks presents a daunting challenge; one that might seem likely to require years or decades of intensive field research.

Rather than a head-on assault of in vivo studies or an endless cycle of laboratory research, Sage represents a third path to understanding IA cognition. Sage is intended to be a scaled world (Ehret, Gray, \& Kirschenbaum, 2000; Gray, 2002) that focuses on a subset of the functional relationships found in the IA's task environment. Like all scaled worlds (Gray, 2002), Sage seeks to preserve the functional relationships of research interest while paring away others.

Key to the Sage scaled world are a series of scenarios developed by and for the intelligence community. Although diverse and heterogeneous, one thing that IAs have in common is their love of historical intelligence case studies and their enjoyment of working through intelligence scenarios developed as training exercises or as intellectual puzzles.

Sage will incorporate these scenarios into what can be considered a sophisticated role playing game for the experienced IA. Indeed, to some degree, Sage will 
resemble the best of today's role-playing games. Built by the renowned virtual world builders of Planet 9 Studios, Sage will resemble a sophisticated blend of WarCraft $^{\mathrm{TM}}$, Civilization ${ }^{\mathrm{TM}}$, and SimCity ${ }^{\mathrm{TM}}$. The end result will be a world, or a scaled world, in which the quest given to the IA will be similar to the tasks given the same agent in daily life. As with all role-playing games, time will be a commodity. It takes time for the IA to acquire information, and to develop and test hypotheses. Unfortunately, the longer the IA takes to do their job, the more likely their solution will be outdated and irrelevant. Hence, the IA's goal is to derive the best solution possible in a limited amount of time. The main differences between Sage and the IA's real world are that the pace will be faster, the penalty for failure much lower, and the same scenario can be played with a wide variety of new tools and techniques. Of not incidental importance, everything about Sage can be instrumented and studied so as to better understand the IA's cognitive processes and how the nature of the task and the nature of the tools available for accomplishing the task influence those processes.

By itself, Sage would be a great game that IAs would enjoy playing and that might help them to sharpen their analytic skills. However, as the discussion of the next four powerful ideas makes clear, Sage is much more than that.

\section{Powerful Idea \#2: Tractability}

A task environment is tractable to researchers if it enables them to address the research question of interest by collecting the data they need with the frequency and accuracy that they need it (Gray, 2002). Sage will provide a tractable environment to two classes of researchers. Those interested in understanding the cognitive processes of IA problem solving and those interested in how new tools and interfaces augment or detract IA problem solving.

Sage has a number of features that make it a tractable research environment. First, since Sage is a scaled world, we know all of the information that is in the Sage environment and all of the ways of accessing that information. Second, all interactive objects and interactive devices are instrumented such that we will collect action log files of every IA action. These log files will be time stamped to the nearest $0.1666 \mathrm{~s}$ and will include information that is complete enough for us to recreate what each IA did and saw from second to second. Third, Sage will be built with eye data in mind. Detailed action traces of eye movements, such as what areas of the screen the IA was studying, where they were looking, how long, etc. are increasingly important to cognitive research. Sage is being built to facilitate the interpretation of eye data. For example, not only will we get a list of raw eye movement data, but these data will be parsed into fixations on and saccades between various screen objects. Fourth, unlike the IA's real world, many different IAs can solve the same Sage scenario. This enables an important element of experimental control. Although the IAs may be heterogeneous, at least the task environment can be controlled.

These first four elements make Sage a lean, mean, and tractable data collection machine. Such elements might suffice if we were merely interested in studying the IA's cognitive processes. However, the intent of Sage is to go beyond understanding problem solving to providing a means of evaluating how interface design affects problem solving and how different tools impact task success. Furthermore, we wish to make such assessments not only by using humans as subjects, but to automate the process by building simulated Cyborgs ( $\operatorname{simBorgs}$ ). These simBorgs will permit us to compare and contrast the usability and effectiveness of hundreds of task environments. Although these features of Sage may be considered elements of tractability, they are powerful ideas in their own right and will be elaborated on below.

\section{Powerful Idea \#3: Sage as an Integrated Task Environment}

Although everyone complains about bad interfaces, all too often poor design is considered a minor nuisance and not a major source of lost productivity and human error. For interactive behavior, milliseconds matter (Gray \& Boehm-Davis, 2000). Human cognition seems designed to optimize its operating costs by finding least-effort combinations of cognition, perception, and action at the $1 / 3$ of a sec level of analysis. Such tradeoffs among microstrategies are not deliberate (i.e., they are typically inaccessible to consciousness), but are real. For example, Gray and $\mathrm{Fu}(2001 ; 2003)$ have shown that small changes in the cost of information acquisition lead people to rely more on knowledge in-the-head and less on knowledge in-the-world with a concomitant increase in errors and time on task. Indeed, even in a simple building blocks task, small changes in the cost of information acquisition change the nature of the task from predominately perceptual-motor to memory-based (Fu \& Gray, 2000).

To make matters worse, task environments usually encompass more than one interface. People do not just use a word processor; along with the word processor they use spreadsheets, web browsers, email systems, electronic calendars, digital cameras, and more. The sum 
total of their electronic and non-electronic tool kits constitutes a complex task environment with multiple, non-complementary interfaces. An emerging view (Fu \& Gray, 2003) is that the inefficient performance that is often reported of users in studies of one task with one interface (Carroll \& Rosson, 1987) is the result of cognition attempting to optimize its microstrategies and methods across the entire task environment. By this view, it is in working between tools in a task environment where the real issues of interface design arise. Interactive behavior that produces efficient performance with one tool with one interface may translate into disastrous and error prone performance when that tool is used in an unintegrated task environment.

Sage will provide a task environment where novel interface designs can be tried and their impact on total task performance can be measured. The use of simBorgs will enable the rapid evaluation of hundred's of combinations of design alternatives. When these alternatives are winnowed down, traditional usability tests with human IAs can be conducted to select the best.

\section{Powerful Idea \#4: Sage as an environment to test new tools}

Traditionally, research issues for information technologies (such as the IA's task) have focused on new tools for data searching, sorting, and processing. These tools will not be neglected by Sage. Indeed, the constricted nature of the scenario-based approach to building Sage will enable the use of here-to-fore experimental, research-oriented formal logic engines such as Oscar (Pollock, 1995), to build an intelligence agent hypothesis test and generation $\left(\mathrm{A}_{\mathrm{HTG}}\right)$ system. Although such tools are not considered to be ready for large, unconstrained data environments, their use in Sage will enable us to access whether they add value to the problem solving process and, just as importantly, whether they will be used by IAs.

The $\mathrm{A}_{\mathrm{HTG}}$ module is the first, not the last tool that we expect to build. However, beyond our own suite of tools, Sage will provide an environment in which some of the tools built by the other 16 projects can be tested in a simple environment with established benchmarks. Indeed, as discussed above, the same tool can be implemented with a variety of interfaces to determine whether its utility increases as its methods for interactive behavior become better matched to the overall task environment.

\section{Powerful Idea \#5: Simulated Cyborgs}

Cyborgs are science-fiction creations that are part human and part machine. An important part of our effort will be to build simBorgs that play the Sage scenarios as well as human IAs. Like the sci-fi cyborgs, our simBorgs combine human and machine components. The simBorgs will be implemented in the ACT-R cognitive architecture (Anderson \& Lebiere, 1998) and will consist of three conceptually different modules; interactive behavior, knowledge engineering, and machine reasoning.

The interactive behavior module will interact directly with Sage much in the manner that IAs interact with Sage. This is the level of highest cognitive fidelity and represents an off-the-shelf use of ACT-R 5.0 (Anderson, Bothell, Byrne, \& Lebiere, 2002). At this level a series of serial and parallel cognitive, perceptual, and action operations are orchestrated, at the $50-\mathrm{msec}$ level, to produce the non-deliberative, least-effort tradeoffs that determine, for example, whether a fact is verified by comparison to what is held in memory or by a visual search and reacquisition of the information from the computer screen.

Like the IAs, the interactive behavior module will interact differently with different scenarios. It will also interact differently with the same scenario when run with different configurations of tools (i.e., different task environments). Having a family of cognitively realistic models of interactive behavior will enable us to quickly predict the influence that various "designed environments" have on the IA's patterns of success and failure.

Success at designing better task environments for IAs requires more than models that interact with their environment the way that IAs interact. The models' activities must be directed towards the same information acquisition goals as the IAs and the information collected must be used for hypothesis generation and testing much like the IAs. These requirements strain the limits of current computational cognitive modeling. Although modern cognitive science understands much about expert reasoning, complete and cognitively plausible models of expert reasoning are research projects unto themselves. At present, developing the families of models required to account for the range of IA reasoning in multiple Sage scenarios would greatly exceed the time and resources available for this project.

To finesse this problem, the knowledge engineering module guides interactive behavior using sets of scenario-specific schemas. These schemas are derived by knowledge engineering techniques (primarily protocol analysis) from expert IAs as they solve the Sage scenarios. The schemas will guide information acquisition activities and cache the results. (Developing these scenario-specific schemas is, in itself, a major 
knowledge engineering effort.)

Filled or partially filled schemas will feed into the machine reasoning module. For Sage, we are using the $\mathrm{A}_{\text {HTG }}$ algorithms developed by the Rensselaer Artificial Intelligence and Reasoning (RAIR) lab. The specific algorithms used will be developed in conjunction with input from Booz Allen's knowledge engineers once the set of scenarios has been specified.

The need to support $\mathrm{A}_{\mathrm{HTG}}$ will require us to extend ACT-R by the addition of a black box buffer. In operation, this buffer will be similar to the other buffers of ACT-R 5.0 (e.g., motor, visual attention, and memory retrieval buffers). Indeed, to the extent that the established ACT-R buffers are grounded in neurocognitive data (Anderson et al., 2002), the addition of a buffer enabling the sending and receiving of information to an $\mathrm{A}_{\mathrm{HTG}}$ module can be conceptualized as a simulated brain implant.

\section{Summary and Conclusions: 5 powerful ideas. Have we promised too much?}

Sage is a scaled world that abstracts key functional relationships from the IA's current task environment and places them into what we view as a prototype of the first integrated task environment for IAs. The first powerful idea, building a scaled world of the IA's task environment, is easy to achieve. The IA community has told us that they enjoy case studies and like the challenge provided by publicly distributed intelligence scenarios. These scenarios are viewed as containing the intellectually challenging part of their job abstracted away from the tedium of long waits for requested information, and other such realities of daily existence. The second powerful idea, building an integrated task environment, is the job of the master world builders at Planet 9 Studios. Planet 9 Studios has much experience building virtual worlds for the Gaming and Defense industries. We have every confidence that the world they build for Sage will look sharp, be innovative, and be fully integrated across task applications. As such, the Sage scaled world can be regarded as a prototype integrated task environment for IAs.

The tractability of Sage for the research questions of current interest is another powerful idea. The reason why most simulation software is so intractable for research purposes is that it is not built with data collection in mind. In Sage, data collection is a fundamental requirement and as such is considered in each design stage. Hence, this powerful idea also seems technically feasible.

While the powerful ideas contained in the notions of scaled worlds, integrated task environment, and research tractable environments do not entail any breakthroughs in technology or science, they do present challenging engineering applications of what we already know how to do.

The fourth powerful idea, using Sage as an environment in which to test our own and other people's new tools also seems doable. Its main complexity stems from the requirement to work with software developers from multiple organizations. The truly difficult part of this idea is in promulgating a set of interface standards that permit other people's software to run as a fully integrated and tractable member of the Sage task environment.

The fifth powerful idea, the building of simBorgs, is a bold and challenging extension of the current state of the art in computational cognitive modeling. SimBorgs represent a marriage of artificial intelligence algorithms to a high-fidelity embodied cognition capable of interacting with a variety of interface designs and making the same least-effort tradeoffs as human users.

All in all the challenge posed by Sage is the right challenge at the right time for the tools and technologies available to the human factors community. In building Sage we expect that progress will be made and that new challenges will be found. In this sense, Sage represents an attempt to advance the state of the art in a demanding context in which any advance is welcomed and valued.

\section{ACKNOWLEDGEMENTS}

Support for the writing of this paper was provided by the Advanced Research and Development Agency through Booz Allen Hamilton to Rensselaer Polytechnic Institute.

\section{REFERENCES}

Anderson, J. R., Bothell, D., Byrne, M. D., \& Lebiere, C. (2002). An integrated theory of the mind. Retrieved October 17, 2002, from http://actr.psy.cmu.edu/papers/403/IntegratedTheory.pdf

Anderson, J. R., \& Lebiere, C. (Eds.). (1998). Atomic components of thought. Hillsdale, NJ: Lawrence Erlbaum Associates.

Brehmer, B., \& Dörner, D. (1993). Experiments with computer-simulated microworlds: Escaping both the narrow straits of the laboratory and the deep blue sea of the field study. Computers in Human Behavior, 9(23), 171-184.

Carroll, J. M., \& Rosson, M. B. (1987). Paradox of the active user. In J. M. Carroll (Ed.), Interfacing thought: Cognitive aspects of human-computer interaction. Cambridge, MA: MIT Press.

Ehret, B. D., Gray, W. D., \& Kirschenbaum, S. S. 
(2000). Contending with complexity: Developing and using a scaled world in applied cognitive research.

Human Factors, 42(1), 8-23.

Fu, W.-t., \& Gray, W. D. (2000). Memory versus Perceptual-Motor Tradeoffs in a Blocks World Task.

In L. R. Gleitman \& A. K. Joshi (Eds.), Twenty-second Annual Conference of the Cognitive Science Society (pp. 154-159). Hillsdale, NJ: Lawrence Erlbaum Associates.

Fu, W.-t., \& Gray, W. D. (2003). Resolving the paradox of the active learner. Manuscript submitted for publication.

Gray, W. D. (2002). Simulated task environments: The role of high-fidelity simulations, scaled worlds, synthetic environments, and microworlds in basic and applied cognitive research. Cognitive Science
Quarterly, 2(2), 205-227.

Gray, W. D., \& Boehm-Davis, D. A. (2000). Milliseconds Matter: An introduction to microstrategies and to their use in describing and predicting interactive behavior. Journal of Experimental Psychology: Applied, 6(4), 322-335.

Gray, W. D., \& Fu, W.-t. (2001). Ignoring perfect knowledge in-the-world for imperfect knowledge inthe-head: Implications of rational analysis for interface design. CHI Letters, 3(1), 112-119.

Gray, W. D., \& Fu, W.-t. (2003). Soft constraints in interactive behavior: The case of ignoring perfect knowledge in-the-world for imperfect knowledge inthe-head. Manuscript submitted for publication.

Pollock, J. L. (1995). Cognitive carpentry: Bradford/MIT Press. 